\author{
N. Sakhraoui, S. Metallaoui \& A. Chefrour
}

\title{
Naturalisation d'Anredera cordifolia (Basellaceae) en Algérie
}

\begin{abstract}
Sakhraoui, N., Metallaoui, S. \& Chefrour, A.: Naturalisation d'Anredera cordifolia (Basellaceae) en Algérie. — Fl. Medit. 29: 159-162. 2019. — ISSN: 1120-4052 printed, 22404538 online.

The occurrence and actual status of Anredera cordifolia (Basellaceae) in Algeria is here reported, for the first time. The species has been recorded in many localities within Skikda area. It is the only representative of Basellaceae in the whole country. Maintained in observation area within the native flora for many years, the plant could be considered as naturalized.
\end{abstract}

Key words: Boussingaultia cordifolia, alien species, degree of naturalization, Algeria.

\section{Introduction}

En période coloniale, l'Algérie a connu une introduction massive de matériel végétal pour des fins alimentaire, commerciale ou ornementale. En 1867, Hardy, le directeur du jardin d'essai du Hamma, dénombre 8214 espèces et variétés (Carra \& Gueit 1952) dont plusieurs sont aujourd'hui naturalisées ou presque dans les différentes régions du pays (Meddour \& al. 2016) avec un probable devenir envahissant. Les espèces envahissantes constituent un véritable danger pour la biodiversité via la réduction de la richesse spécifique autochtone (Vilà \& al. 2015), la pollution génétique et l'érosion génétique. Elles sont d'ailleurs, considérées comme la deuxième cause d'érosion de la biodiversité après la destruction et la fragmentation des habitats (OTA 1993).

Au cours de la réalisation de prospections botaniques dans la wilaya de Skikda, située au NE Algérien (Sakhraoui \& al. 2019), nous avons noté la présence d'une espèce exotique à léger potentiel envahissant dont l'existence et le statut en Algérie n'ont jamais été rapportés, il s'agit de la liane de Madère, Anredera cordifolia (Ten.) Steenis. Les relevés floristiques que nous avons réalisés tentent essentiellement à mettre le point sur les différents milieux colonisés dans un but à envisager des stratégies de lutte contre son éventuelle extension. Certaines caractéristiques biologiques observées in situ ont été notées (période de floraison, aptitude à la fructification et modalité de reproduction végétative). L'appartenance taxonomique, l'origine et la date d'introduction de cette espèce ont été établies à partir de la littérature. Le type biologique a été déterminé selon Raunkiaer (1934) 
et le statut d'indigénat selon Magnanon \& al. (2008). Les spécimens récoltés ont été déposés dans l'herbier de la wilaya maintenu au niveau du laboratoire de botanique du département des sciences de la nature et de la vie de l'Université de Skikda.

\section{Description, répartition géographique et degré de naturalisation/statut actuel}

Anredera cordifolia (Ten.) Steenis, Fl. Malesiana, Ser.1, Spermatoph. 5(3): 303 (1957) (=Boussingaultia cordifolia Ten. in Ann. Sci. Nat., Bot. sér. 3, 19: 355 (1853)).

A. cordifolia communément appelée liane de Madère, est une herbe à gros tubercule souterrain, à tiges volubiles. Feuilles alternes cordiformes ou ovales un peu charnues, entières. Les fleurs sont petites, blanches disposées en grappes terminales et axillaires (Maire 1962). La plante est une géophyte, originaire d'Amérique du Sud (Wagner \& al. 1999), qui a été introduite en Algérie entre 1842 et 1867 par le jardin d'essai du Hamma (Carra \& Gueit 1952).

Elle a également été introduite dans de nombreux pays en tant que plante d'ornement où elle est devenue envahissante notamment en Australie, Afrique du Sud, Nouvelle Zélande et Hawaï (Weber 2017). En méditerranée, la liane de madère est naturalisée en Italie (Galasso \& al. 2018), en Grèce (Arianoutsou \& al. 2010) et en Croatie (Stancic \& Mihelj 2010), alors qu'elle est considérée comme envahissante avérée en Espagne (Capdevilla Argüelles \& al. 2006). Dans les zones d'introduction, l'espèce semble se reproduire uniquement par voie végétative (Stancic \& Mihelj 2010 ; Kottaimuthu \& al. 2011), les nombreux tubercules aériens accrochés aux tiges semblent jouer un rôle important dans la propagation rapide de l'espèce. Ces derniers peuvent se détacher facilement et rester viables de 5 à 10 ans (Vivian-Smith \& al. 2007). D'après Kolar \& Lodge (2001), les probabilités d'envahissement augmentent si la plante possède des antécédents d'envahissement et une reproduction végétative.

En Afrique du Nord, sa présence comme plante d'ornement a été rapportée par Maire (1962) sans indication précise des pays dans lesquels elle a été cultivée. Plus récemment, la plante est rapportée naturalisée au Maroc (Uotila 2009; APD 2019) alors qu'en Algérie elle n'a jamais été signalée naturalisée (Quézel \& Santa 1962; 1963; Dobignard \& Chatelain 2011).

En Algérie, et plus précisément dans notre zone d'étude, cette espèce a été retrouvée en 2014 dans plusieurs points des cités Saleh Chebel (commune de Hamadi Krouma) et Larbi Ben M'Hidi (commune de Skikda), notamment à la lisière des jardins d'où elle a tendance à échapper. A la cité Larbi Ben M'Hidi, une grande population a été observée au bord de la route du poste 3 dans un terrain abandonné où elle pousse horizontalement, formant un tapis étendu et verticalement en s'accrochant à divers arbustes avoisinants (Pistacia lentiscus, Acacia karoo, Ephedra fragilis, etc.). Ce point de prolifération, situé à quelques mètres du maquis arborescent à Quercus coccifera, Juniperus phoenicea et Chamaerops humilis qui caractérise la dune littorale, est connu d'après le témoignage des habitants depuis au moins 11 années. Elle a également été repérée, en 2015, sur le djebel Mouadher à l'Est de la ville de Skikda en face de la zone industrielle, où elle occupe une surface plus importante et y est représentée par une population plus dense. Les oliviers proliférant sur place lui servent de tuteurs. Dans les différentes localités, la floraison a été observée à partir du 
mois de Juin jusqu'au mois d'Octobre de 2014 à 2018. Les fruits, par contre, n'ont pas été récoltés. La plante peut être considérée comme naturalisée dans la région de Skikda, puisqu'elle se maintient dans l'une des zones d'observation depuis au moins 11 ans et fait part de la flore indigène (Magnanon $\&$ al. 2008). A. cordifolia devrait être surveillée pour éviter qu'elle ne devienne envahissante avérée dans les prochaines années.

\section{Références}

APD (African Plant Database) 2019: Anredera cordifolia (Ten.) Steenis: Conservatoire et Jardin botaniques; Pretoria: South African National Biodiversity Institute. - Published at http://www.villege.ch/musinfo/bd/cjb/africa/details.php?langue $=$ an\&id=23817 [Last Accessed 26 June 2019].

Arianoutsou, M., Bazos, I., Delipetrou, P. \& Kokkoris, Y. 2010: The alien flora of Greece: Taxonomy, life traits and habitat preferences. - Biol. Inv. 12: 3525-3549. https://doi.org/10.1007/s10530010-9749-0

Capdevilla Argüelles, L., Iglesias Garcia, A., Orueta, J.F. \& Zilleti, B. 2006 : Especies exoticas invasoras : Dignostico y bases para la prevencion y el manejo. Série Técnica. - Madrid.

Carra, P. \& Gueit, M. 1952: Le jardin d'essai du Hamma. - Alger.

Dobignard, A. \& Chatelain, C. 2011: Index synonymique de la flore de l'Afrique du Nord, 3. Genève.

Galasso, G., Conti, F., Peruzzi, L., Ardenghi, N. M. G., Banfi, E., Celesti-Grapow, L., Albano, A., Alessandrini, A., Bacchetta, G., Ballelli, S., Bandini Mazzanti, M., Barberis, G., Bernardo, L., Blasi, C., Bouvet, D., Bovio, M., Cecchi, L., Del Guacchio, E., Domina, G., Fascetti, S., Gallo, L., Gubellini, L., Guiggi, A., Iamonico, D., Iberite, M., Jiménez-Mejías, P., Lattanzi, E., Marchetti, D., Martinetto, E., Masin, R. R., Medagli, P., Passalacqua, N. G., Peccenini, S., Pennesi, R., Pierini, B., Podda, L., Poldini, L., Prosser, F., Raimondo, F. M., Roma-Marzio, F., Rosati, L., Santangelo, A., Scoppola, A., Scortegagna, S., Selvaggi, A., Selvi, F., Soldano, A., Stinca, A., Wagensommer, R. P., Wilhalm, T. \& Bartolucci, F. 2018: An updated checklist of the vascular flora alien to Italy. - Pl. Biosyst. 152(3): 556-592. https://doi.org/10.1080/11263504.2018.1441197

Kolar, C. S. \& Lodge, D. M. 2001: Progress in invasion biology: Predicting invaders. - Trends Ecol. Evol. 16(4): 199-204. https://doi.org/10.1016/S0169-5347(01)02101-2

Kottumuthu, R., Ganesan, R. \& Vijayan, R. 2011: Anredera cordifolia (Tenore) Steenis (Basellaceae) - a new record for India. - Bio. Diver. 40: 5517- 5518.

Magnanon, S., Geslin, J., Lacroix, P. \& Zambettakis, C. 2008 : Examen du statut d'indigénat et du caractère invasif des plantes vasculaires de basse Normandie, Bretagne et pays de la Loire. Proposition d'une première liste de plantes invasives et potentiellement invasives pour ces régions. Bulletin du Conservatoire Botanique National de Brest. - E.R.I.C.A. 21: 73-104.

Maire, R. 1962 : Flore de l'Afrique du Nord, Dicotyledonae. - Paris.

Meddour, R. El Mokni, R. \& Vela E. 2016 : État de l'art sur les plantes envahissantes ou à caractère invasif introduites en Algérie et en Tunisie. XV OPTIMA Meeting, 6-11 June 2016. Montpellier.

OTA. 1993: Harmful non-indigenous species in the United States. Office of Technology and Assessment. - United States.

Quézel, P. \& Santa, S. 1962 : Nouvelle flore d'Algérie et des régions désertiques méridionales, 1. Paris.

— \& - 1963: Nouvelle flore d'Algérie et des régions désertiques méridionales, 2. - Paris.

Raunkiaer, C. 1934: The life forms of plants and statistical plant, Geography. - Oxford. 
Sakhraoui, N., Metallaoui, S., Chefrour, A. \& Hadef, A. 2019: La flore exotique potentiellement envahissante d'Algérie : première description des espèces cultivées en pépinières et dans les jardins. - Biotechnol. Agron. Soc. Environ. 23 (2): 63-73. https://doi.org/10.25518/17804507.17902

Stancic, Z. \& Mihelj, D. 2010: Anredera cordifolia (Ten.) Steenis (Basellaceae), naturalised in south Croatia. - Nat. Croat. 19(1): 273-279.

Uotila, P. 2009: Basellaceae. - In: Euro+Med Plantbase - the information resource for EuroMediterranean plant diversity. at http://ww2.bgbm.org/EuroPlusMed/PTaxonDetail.asp?NameId=47683\&PTRefFk=7300000. [Last Accessed 26 June 2019].

Vilà, M., Rohr, R. P., Espinar, J. L., Hulme, P.E., Pergl, J., Le Roux, J. J., Schaffner, U. \& Pyšeket, P. 2015: Explaining the variation in impacts of non-native plants on local-scale species richness: the role of phylogenetic relatedness. - Glob. Ecol. Biogeogr. 24: 139-146. https://doi.org/10.1111/geb.1224

Vivian-Smith, G., Lawson, B.E., Turnbull, I. \& Downey, P. O. 2007: the biology of Australian weeds.46. Anredera cordifolia (Ten) Steenis. - Plant Prot. Q. 22(1): 1-10.

Wagner, W. L., Herbst, D. R. \& Sohmer, S.H. 1999: Manual of the Flowering Plants of Hawaï, 1-2. - Honolulu.

Weber, E. 2017: Invasive plant species of the world: A reference guide to environmental weeds, 2e ed. - Boston.

Adresses des auteurs :

Nora Sakhraoui ${ }^{1 *}$, Sophia Metallaoui ${ }^{1}$ et Azzedine Chefrour ${ }^{2}$,

$1 *$ Département des Sciences de la Nature et de la Vie (SNV). Faculté des sciences. Université 20 Aout 1955, BP 26 Route d'El-Hadaiek, Skikda, 21000, Algérie. Email: sakhraouinora05@gmail.com; metallaoui_s@yahoo.fr

${ }^{2}$ Département de Biologie. Faculté des Sciences de la Nature et de la Vie. Université Mohamed Cherif Messaadia, Souk Ahras, 41000, Algérie. E-mail: azchefrour@yahoou.fr

*Auteur correspondant 